\title{
ON THE NON-EXISTENCE OF $L^{2}$ SOLUTIONS OF A CLASS OF NON-LINEAR DIFFERENTIAL EQUATIONS $\dagger$
}

\author{
by J. BURLAK \\ (Received 2nd October 1964)
}

\section{Introduction}

In 1950, Wintner (11) showed that if the function $f(x)$ is continuous on the half-line $[0, \infty)$ and, in a certain sense, is " small when $x$ is large" then the differential equation

$$
y^{\prime \prime}+(x) y=0
$$

does not have $L^{2}$ solutions, where the function $y(x)$ satisfying (l) is called an $L^{2}$ solution if

$$
0<\int_{0}^{\infty}|y(x)|^{2} d x<\infty
$$

More precisely, he proved

Theorem 1. The differential equation (1) cannot have an $L^{2}$ solution if

$$
\int_{0}^{\infty} x^{3}|f(x)|^{2} d x<\infty
$$

where $\limsup _{x \rightarrow \infty}|f(x)| \neq 0$ is allowed.

We shall prove a number of analogues of this result for the nonlinear differential equation

$$
y^{\prime \prime}+f(x) g(y)=0 .
$$

Our simplest result, involving the fewest assumptions about the functions $f(x)$ and $g(y)$, is Theorem 5, which is stated in full in $\S 5$. However, our most detailed and explicit result, with reference to which it is convenient to discuss all the others, is

Theorem 2. Suppose that the functions $f(x)$ and $g(y)$ satisfy the following conditions:

(i) $f(x)$ is continuous on $[0, \infty)$;

(ii) $g(y)$ is continuous on $(-\infty, \infty)$, satisfies the condition

$$
\left|g\left(y_{1}\right)-g\left(y_{2}\right)\right| \leqq L\left|y_{1}-y_{2}\right|
$$

$\dagger$ This research was carried out at Duke University and was supported by the United States Air Force under grant AF-AFOSR 435-63, monitored by the Air Force Office of Scientific Research.

E.M.S.-S 
for any $y_{1}$ and $y_{2}$, where $L$ is a constant which is independent of $y_{1}$ and $y_{2}$ over any bounded interval, and satisfies the condition

$$
g(y)=O(|y|) \text { as }|y| \rightarrow \infty
$$

(iii) if the continuous function $y(x)$ tends to a non-zero limit as $x \rightarrow \infty$ then $\int_{0}^{\infty}|g\{y(x)\}|^{2} d x$ is divergent;

(iv) if $\int_{0}^{\infty}|y(x)|^{2} d x<\infty$ (cf. condition (iv $)$ in $\left.\S 2\right)$ then there exist constants $M$ and $T, 0<M<\infty, 0 \leqq T<\infty$, such that

$$
\int_{t}^{\infty}|g\{y(x)\}|^{2} d x \leqq M \int_{t}^{\infty}|y(x)|^{2} d x \text { for all } t \geqq T .
$$

Then the differential equation (4) cannot have a solution $y(x)$ for which

$$
\int_{0}^{\infty}|y(x)|^{2} d x>0 \text { and } \int_{0}^{\infty}|g\{y(x)\}|^{2} d x<\infty
$$

if $f(x)$ satisfies (3).

Although the conditions of Theorem 2 (which ensure, among other things, that every solution of (4) exists on the whole half-line $[0, \infty)$ ) appear to be complicated and rather stringent, we have not in fact given our result in its full generality, to avoid complicating the statement of the theorem still further. Instead, we have interpolated an analysis of the conditions of the theorem in $\S 2$, indicating both the purpose of the different conditions and the extent to which they may be relaxed. This analysis is also used in $\S \mathbf{5}$.

The proof of Theorem 2 is divided into two parts, some preliminary results being isolated in $\S 3$ and the main part of the proof being given in $\S \mathbf{4}$, where we also state a corollary referring to $L^{2}$ solutions of (4). Finally, in $\S 5$, we make some of the discussion in $\S 2$ more precise by first restating Theorem 2 under more general conditions than those above and then considering some special cases, where we relax some of the conditions on $f(x)$ and $g(y)$ (and no longer insist on every solution of (4) being defined on the whole half-line $[0, \infty)$ ).

It is a pleasure to conclude this introduction by acknowledging a number of useful comments by Professor J. J. Gergen.

\section{The conditions of Theorem 2}

First we observe that the conditions of Theorem 2 are certainly satisfied by any function $g(y)$ which is differentiable on $(-\infty, \infty)$ and for which there exist constants $a>0, A>0$ such that $a|y| \leqq|g(y)| \leqq A|y|$ for all $y$ (for example, by the functions $y e^{\sin y}$ and $y(a \cos y+b \sin y+c)$ for $\left.c^{2}>a^{2}+b^{2}\right)$. Also, it is easy to verify that the conditions are satisfied by such functions as $y^{3} /\left(1+y^{2}\right), \quad y / \sqrt{ }\left(1+y^{2}\right), \quad 1-\left(1+y^{2}\right)^{-\frac{1}{2}}, \quad y\left\{1-\left(1+y^{2}\right)^{-\frac{1}{t}}\right\}, \quad \tanh y, 1-e^{-|y|}$, 
$y\left(1-e^{-y^{2}}\right)$ and, indeed, by any non-decreasing function of the form $g(y)=y h(y)$ with $h(y)$ continuous and bounded.

The last condition (iv) means, essentially, that if $y(x)$ is square-integrable on $[0, \infty)$ then so is $g\{y(x)\}$; it also ensures that $g(0)=0$ (cf. $\S 4)$. The condition (iii) means, essentially, that $g\{y(x)\}$ fails to be square-integrable on $[0, \infty)$ when $y(x)$ fails drastically to be square-integrable on $[0, \infty)$; it also ensures that $g(y) \neq 0$. These conditions are sufficient for our purpose, but it is possible that they can be improved: as we shall see in the proof of Lemma 1, the condition (iv) can certainly be replaced by the weaker condition

(iv') (7) holds for any function $y(x)$ such that $\int_{0}^{\infty}|y(x)|^{2} d x<\infty$ and $y(x) \rightarrow 0$ as $x \rightarrow \infty$.

On the other hand the condition (3) on $f(x)$ cannot be improved without further conditions on $g(y)$. For, as was pointed out by Wintner (11) in the case of equation (1), where $g(y)=y$, the condition (3) is best possible in the sense that it cannot be replaced by the two conditions

$$
\int_{0}^{\infty} x^{3-\varepsilon}|f(x)|^{2} d x<\infty \text { and } \int_{0}^{\infty} x^{3}|f(x)|^{2+\varepsilon} d x<\infty
$$

with $\varepsilon>0$, since these conditions are satisfied for all $\varepsilon>0$ by the function $f(x)=\left\{\begin{array}{l}b, 0 \leqq x \leqq 1 \\ b / x^{2}, x \geqq 1\end{array}\right.$, and then (1) has a solution which is of the form $y(x)=x^{-a(b)}$ for $x \geqq 1$ (and is continuously differentiable for $0 \leqq x \leqq 1$ ), where $a(b)=\frac{1}{2}\{-1+\sqrt{(}(1-4 b)\}$ can be chosen to be arbitrarily large by the appropriate choice of $b$. This is the reason for our putting condition (3) at the end of the statement of the theorem, separately from the above conditions: the conditions we have mentioned above are the ones that really characterise our problem, but it is condition (3) that is crucial.

The remaining conditions are less important, in the sense that they are less definitive, being concerned merely with the existence and uniqueness of solutions of (4). Thus, the continuity of $f(x)$ and $g(y)$ guarantees the local existence of twice continuously differentiable solutions of (4) (cf. for example (1, pp. 26, 34)) and the condition (5) is easily seen to imply that the first-order system

$$
z^{\prime}=f(x) g(y), y^{\prime}=z,
$$

equivalent to (4), satisfies the Lipschitz condition

$$
\max \left\{\left|f(x) g\left(y_{1}\right)-f(x) g\left(y_{2}\right)\right|,\left|z_{1}-z_{2}\right|\right\} \leqq K \max \left\{\left|y_{1}-y_{2}\right|,\left|z_{1}-z_{2}\right|\right\},
$$

where $K$ is a constant, independent of $y_{1}, y_{2}, z_{1}$ and $z_{2}$ in any bounded region; this guarantees uniqueness of the solution to the initial-value problem for (4) (cf. (1, pp. 37, 38)). Further, the condition (6), together with the continuity of $f(x)$, ensures that every solution of (4) exists for all $x$ on the half-line $[0, \infty)$ and not merely locally: if $f(x)$ is bounded as $x \rightarrow \infty$ this follows by a well-known criterion of Wintner (9, p. 279); if $f(x)$ is not bounded as $x \rightarrow \infty$ then we can 
appeal to a more general criterion (cf. (12) below) due to Conti (3) (strictly speaking, Conti's criterion is needed to give a posteriori justification of Theorem 1 in the case when $f(x)$ is unbounded as $x \rightarrow \infty)$. A non-local existence theorem of this kind is obviously essential if Theorem 2 is not to be vacuous. However, as we shall see below and in $\S \mathbf{5}$, we do not need to demand that every solution of (4) be defined on $[0, \infty)$.

The conditions of the last paragraph can be relaxed to some extent. Thus, the condition (5) implying the Lipschitz condition (10) may be replaced by a weaker (but more complicated) condition implying that the equivalent system (9) satisfies one of a number of conditions of the form

$\max \left\{\left|f(x) g\left(y_{1}\right)-f(x) g\left(y_{2}\right)\right|,\left|z_{1}-z_{2}\right|\right\} \leqq K(x) F\left[\max \left\{\left|y_{1}-y_{2}\right|,\left|z_{1}-z_{2}\right|\right\}\right]$

which guarantee uniqueness of the solution to the initial-value problem for (4); the particular uniqueness criteria of Osgood, Nagumo, Tonelli, LaSalle and others (cf. (1, pp. 40, 42, 70), (7, pp. 98-113)) are obtained by appropriate choices of the functions $K$ and $F$. It is worth remarking here that, with the functions $F$ that have been used so far, the condition (11) (and, hence, (5)) necessarily implies the continuity of $g(y)$. However, the function $f(x)$ may have a finite number of finite discontinuities: for it is well known that the local existence theorem still yields a continuous solution under these circumstances (cf. (5, p. 66)); also, it is easy to see that the uniqueness criteria, above, and the non-local conditions, below, remain valid and we shall verify, in the proof of Lemma 1, that Theorem 2 still holds. Finally, the condition (6) may be replaced by a condition which ensures that the system (9) satisfies a condition of the form

$$
\left[\{f(x) g(y)\}^{2}+z^{2}\right]^{\frac{1}{2}} \leqq m(x) L\left\{\left(y^{2}+z^{2}\right)^{\frac{1}{2}}\right\}
$$

for all $y, z$ and for $x \geqq x_{0}$, where $x_{0}, 0 \leqq x_{0}<\infty$, is some constant, $m(x)$ is continuous for $x \geqq x_{0}$ and $L(r)$ is a function satisfying $L(r)>0$ for $r>0$ and $\lim _{u \rightarrow \infty} \int_{u_{0}}^{u} d r / L(r)=+\infty$ for some $u_{0}, 0 \leqq u_{0}<\infty$. This is the criterion of Conti referred to above; it includes as special cases the criterion of Wintner referred to above, as well as Wintner's more general criterion $(9$, p. $283 ; 10)$, and guarantees that all solutions of (4) exist on the whole half-line $[0, \infty)$. Still other criteria may be used instead of (12) (cf. (4) (2, p. 4)).

More important perhaps is the following remark. If we are assured $a$ priori that there exists a unique, continuous solution to the initial-value problem for (4) which is necessarily defined for all $x$ on $[0, \infty)$, or if we are dealing with a particular continuous solution $y_{0}(x)$ of (4) which exists on the whole half-line $[0, \infty)$ and is known to be the only solution of (4) satisfying the initial conditions $y(a)=y_{0}(a), y^{\prime}(a)=y_{0}^{\prime}(a), 0 \leqq a<\infty$, then most of the above complication falls away and the only conditions that concern us are (iii), (iv) and the few facts about $f(x)$ and $g(y)$ that are used explicitly in the following proofs. This remark will be made more precise in $\S 5$. 


\section{Some preliminary results}

Lemma 1. If the conditions of Theorem 2 are satisfied, except that (3) is replaced by the condition $\int_{0}^{\infty} x^{2}|f(x)|^{2} d x<\infty$, and if (4) has a solution $y(x)$ satisfying (8), then $y(x) \rightarrow 0$ and $y^{\prime}(x) \rightarrow 0$ as $x \rightarrow \infty$.

Proof. We observe first that such solutions do exist, by the remarks on condition (3) in $\S 2$.

Since $f(x)$ and $g(y)$ are continuous it follows from (4) that $y^{\prime \prime}$ exists everywhere and is bounded on every bounded interval and so (cf. $(6$, p. 180), (8, p. 368)), (4) implies that

$$
y^{\prime}(v)-y^{\prime}(u)=-\int_{u}^{v} f(x) g\{y(x)\} d x, u \leqq x \leqq v .
$$

Further

$$
\left|\int_{u}^{v} f(x) g\{v(x)\} d x\right| \leqq\left[\int_{u}^{v}|f(x)|^{2} d x\right]^{\frac{1}{2}}\left[\int_{u}^{v}|g\{y(x)\}|^{2} d x\right]^{\frac{1}{2}},
$$

by the Schwarz inequality and the fact that $f(x)$ is continuous. Moreover, the condition $\int_{0}^{\infty} x^{2}|f(x)|^{2} d x<\infty$ and the fact that, since $f(x)$ is continuous, $\int_{0}^{\delta}|f(x)|^{2} d x<\infty$ for $0<\delta<\infty$ imply that $\int_{0}^{\infty}|f(x)|^{2} d x<\infty$. Hence, by (14) and (8), the right-hand side of (13) tends to zero as $u \rightarrow \infty, v \rightarrow \infty$, i.e. $y^{\prime}(x)$ tends to a limit as $x \rightarrow \infty$. This limit must be zero since $\left|y^{\prime}(\infty)\right| \neq 0$ would imply, by quadrature (which is valid by the same argument as led to (13)) that $|y(x)| \rightarrow \infty$ as $x \rightarrow \infty$. But, by condition (iii) of Theorem 2 , this would contradict (8).

Before continuing, we observe that the above argument holds also when $f(x)$ is continuous except for a finite number of finite discontinuities. For, in this case, $y^{\prime \prime}$ is defined and finite (indeed, continuous) except at a finite number of points and integrable over every bounded interval. Further, since the local existence theorem still holds (cf. $\S 2$ ), the function $z$ in (9) (i.e. the function $\left.y^{\prime}(x)\right)$ is continuous and so we may still write down (13) (cf. (6, p. 183)). Finally, we still have $\int_{u}^{v}|f(x)|^{2} d x<\infty$, so (14) still holds, and $\int_{0}^{\infty}|f(x)|^{2} d x<\infty$, as above, so the rest of the above argument (and, hence, also the proof of Lemma 1) follows without any modification.

We can now deduce from (13) that

$$
y^{\prime}(x)=\int_{x}^{\infty} f(s) g\{y(s)\} d s
$$


i.e. that

$$
\begin{aligned}
\int_{0}^{\infty}\left|y^{\prime}(x)\right| d x & \leqq \int_{0}^{\infty}\left[\int_{x}^{\infty}|f(s) g\{y(s)\}| d s\right] d x \\
& =\int_{0}^{\infty} s|f(s) g\{y(s)\}| d s,
\end{aligned}
$$

where, for the moment, the integrals on the right-hand side may be infinite; (16) shows that the last integral is finite and so we easily justify the interchange of orders of integration (cf. (8, pp. 390-392)). Thus, by the Schwarz inequality and the conditions of the lemma,

$$
\int_{0}^{\infty}\left|y^{\prime}(x)\right| d x \leqq\left[\int_{0}^{\infty} s^{2}|f(s)|^{2} d s\right]^{\frac{1}{2}}\left[\int_{0}^{\infty}|g\{y(s)\}|^{2} d s\right]^{\frac{1}{2}} .
$$

But now (16) and (8) show that $\int_{0}^{\infty}\left|y^{\prime}(x)\right| d x<\infty$, i.e. that $y(x)$ is of bounded total variation. This means that $y(x)$ tends to a limit as $x \rightarrow \infty$ and indeed, that this limit is zero for, by condition (iii) of Theorem $2,|y(\infty)| \neq 0$ would contradict (8).

Lemma 2. Under the conditions of Theorem 2, if $y(x)$ is a solution of (4) satisfying (8) then it is an $L^{2}$ solution.

Proof. By (15), Lemma 1 and the argument used to derive (13),

$$
y(x)=-\int_{x}^{\infty}\left[\int_{u}^{\infty} f(v) g\{y(v)\} d v\right] d u
$$

i.e., as in the proof of Lemma 1, following (15),

Hence, if $t \geqq 0$,

$$
|y(x)| \leqq \int_{x}^{\infty} u|f(u) g\{y(u)\}| d u .
$$

$$
\begin{aligned}
\int_{t}^{\infty}|y(x)|^{2} d x & \leqq \int_{t}^{\infty}\left[\int_{x}^{\infty} u|f(u) g\{y(u)\}| d u\right]^{2} d x \\
& \leqq \int_{t}^{\infty}\left[\int_{x}^{\infty} u^{2}|f(u)|^{2} d u\right]\left[\int_{x}^{\infty}|g\{y(u)\}|^{2} d u\right] d x
\end{aligned}
$$

by the Schwarz inequality and the fact that $\int_{0}^{\infty} u^{2}|f(u)|^{2} d u<\infty$, which follows in the same way as the result $\int_{0}^{\infty}|f(x)|^{2} d x<\infty$ in the proof of Lemma 1. Thus

$$
\int_{t}^{\infty}|y(x)|^{2} d x \leqq \int_{t}^{\infty} x^{3}|f(x)|^{2} d x \cdot \int_{t}^{\infty}|g\{y(x)\}|^{2} d x,
$$

where we have treated the first factor on the right-hand side in the same way 
as we treated an integral in Lemma 1, following (15). But now (17) immediately implies the conclusion of Lemma 2 , since $\int_{0}^{\infty}|y(x)|^{2} d x>0$, by (8).

Although it may appear a little paradoxical to refer in Lemma 2 to a solution which will be seen not to exist once we have proved Theorem 2, it should be remembered that Lemmas 1 and 2 are really an integral part of the proof of Theorem 2 and are not intended as independent results. Thus, Lemma 1 is also valid under the same conditions as Lemma 2; we have preferred to state it under slightly more general conditions above so that it refers to actual functions and can be used as an independent result.

Clearly, Lemmas 1 and 2 do not require all the conditions of Theorem 2 for their validity; we shall return to this point in $\S 5$.

\section{Proof of Theorem 2}

Suppose that the conditions of the theorem are satisfied and that $y(x)$ is a solution of (4) satisfying (8). Then, proceeding as in the proof of Lemma 2, we find, by (17), Lemma 2 and condition (iv) of Theorem 2, that

$$
\begin{aligned}
\int_{t}^{\infty}|y(x)|^{2} d x & \leqq \int_{t}^{\infty} x^{3}|f(x)|^{2} d x \cdot \int_{t}^{\infty}|g\{y(x)\}|^{2} d x \\
& \leqq M \int_{t}^{\infty} x^{3}|f(x)|^{2} d x \cdot \int_{t}^{\infty}|y(x)|^{2} d x
\end{aligned}
$$

for all $t \geqq T$. (If we replace condition (iv) of Theorem 2 by condition (iv') (cf. § 2) then we justify (18) by (17), Lemma 1 and condition (iv').) But now, as we shall see in a moment, $\int_{t}^{\infty}|y(x)|^{2} d x>0$ for all $t \geqq T$ and so (18) implies that

$$
\int_{t}^{\infty} x^{3}|f(x)|^{2} d x>\frac{1}{M}>0
$$

for all $t \geqq T$, where $M$ is independent of $t$. This contradicts (3) as $t \rightarrow \infty$ and so proves the theorem.

It remains to show that $\int_{t}^{\infty}|y(x)|^{2} d x>0$ for all $t \geqq T$. If not, say if $\int_{x_{0}}^{\infty}|y(x)|^{2} d x=0$, then $y(x)=0$ for $x \geqq x_{0}$ i.e. by condition (iv) (or (iv')) of Theorem $2, g\{y(x)\}=0$ for $x \geqq x_{0}$, i.e. $g(0)=0$. It follows that the function $y \equiv 0$ is a solution of (4) and so, by the uniqueness of the solution to the initial-value problem for (4), referred to in $\S 2$, the above solution $y(x)$ satisfies $y(x)=0$ for $x \geqq 0$, which contradicts the condition $\int_{0}^{\infty}|y(x)|^{2} d x>0$ in (8).

An immediate extension of Theorem 2, which explains the title of the paper, is 
Corollary 1. Under the conditions of Theorem 2, equation (4) cannot have $L^{2}$ solutions.

Naturally here, and in the following Corollaries, we do not regard (8) as one of the conditions.

Proof. If $y(x)$ were an $L^{2}$ solution then, by (7) and the fact that

$$
\int_{0}^{T}|g\{y(x)\}|^{2} d x<\infty
$$

(since $g(y)$ and $y(x)$ are continuous), it would follow that $y(x)$ satisfies (8), which would contradict Theorem 2 .

If condition (iv) of Theorem 2 is replaced by condition (iv') (cf. $\S 2$ ) then the above proof fails and the validity of Corollary 1 remains an open problem. However, we clearly do have

Corollary 1a. Under the conditions of Theorem 2 , with condition (iv) replaced by condition (iv'), equation (4) cannot have $L^{2}$ solutions $y(x)$ such that $y(x) \rightarrow 0$ as $x \rightarrow \infty$.

\section{Some extensions of Theorem 2}

As we remarked in $\S 2$, the conditions of Theorem 2 (in particular, the conditions for existence on $[0, \infty)$ and for uniqueness) are sufficient but not necessary. Thus, the statement of the theorem can be simplified (and the result generalised somewhat) if only those conditions that are explicitly used in the proof are explicitly stated, as in

Theorem 3. Suppose that the functions $f(x)$ and $g(y)$ satisfy the following conditions:

(i) $f(x)$ and $g(y)$ are such as to ensure that the initial-value problem for equation (4) has a unique, continuously differentiable solution $y(x)$ which exists on the whole half-line $[0, \infty)$ and for which $y^{\prime \prime}(x)$ is integrable over every bounded interval and finite except at a finite or denumerable set of points;

(ii) there exists a constant $\delta, 0<\delta<\infty$, such that $\int_{0}^{\delta}|f(x)|^{2} d x<\infty$;

(iii) $g(y)$ satisfies conditions (iii) and (iv) of Theorem 2.

Then equation (4) cannot have a solution $y(x)$ for which (8) holds if $f(x)$ satisfies (3).

Although the above theorem is rather more general than Theorem 2 it is easy to verify that the proof of Theorem 2 given in $\S \S 3$ and 4 can be used, without any modification, to establish Theorem 3. Similarly, the proof of Corollary 1 immediately yields

Corollary 2. Under the conditions of Theorem 3, equation (4) cannot have an $L^{2}$ solution $y(x)$ for which $\int_{0}^{T}|g\{y(x)\}|^{2} d x<\infty$ if $f(x)$ satisfies (3). 
As before, Theorem 3 also holds if $g(y)$ is required to satisfy the condition (iv') of Theorem 2 (cf. $\S 2$ ) instead of condition (iv). However, as in the caseof Corollary 1 , the validity of Corollary 2 is then still open and we only have

Corollary 2a. Under the conditions of Theorem 3, with condition (iv) of Theorem 2 replaced by condition (iv $\left.{ }^{\prime}\right)$, equation (4) cannot have an $L^{2}$ solution $y(x)$ such that $\int_{0}^{T}|g\{y(x)\}|^{2} d x<\infty$ and $y \rightarrow 0$ as $x \rightarrow \infty$ if $f(x)$ satisfies (3).

The results we have obtained so far refer to all the solutions of (4), sinceour hypotheses have guaranteed that every solution exists on the whole halfline $[0, \infty)$. However, we can extend these results to more general differential equations if we consider only those solutions which do happen to be defined on the whole half-line without insisting that this be true of every solution. of (4).

Since the proof of Theorem 2 refers to a particular solution and does not rest on those hypotheses which guarantee the non-local existence of solutions, we can immediately deduce the following results. (Theorems 4 and 5 are the rough analogues of Theorems 2 and 3, respectively, Corollary 3 is the analogue of Corollaries 1 and 2, Corollary 3a of Corollaries 1a and 2a).

Theorem 4. Suppose that the functions $f(x)$ and $g(y)$ satisfy the following conditions:

(i) $f(x)$ is continuous on $[0, \infty)$;

(ii) $g(y)$ is continuous on $(-\infty, \infty)$, satisfies the condition (5) and satisfies conditions (iii) and (iv) of Theorem 2;

Then, if $y(x)$ is a solution of (4) which exists on the whole half-line $[0, \infty)$, (8) cannot hold if $f(x)$ satisfies (3).

Theorem 5. Suppose that the functions $f(x)$ and $g(y)$ satisfy the following conditions:

(i) there exists a constant $\delta, 0<\delta<\infty$, such that $\int_{0}^{\delta}|f(x)|^{2} d x<\infty$;

(ii) if the continuous function $y(x)$ tends to a non-zero limit as $x \rightarrow \infty$ then $\int_{0}^{\infty}|g\{y(x)\}|^{2} d x$ is divergent;

(iii) if $\int_{0}^{\infty}|y(x)|^{2} d x<\infty$ (cf. also condition (iv') in § 2) then there exist constants $M$ and $T, 0<M<\infty, 0 \leqq T<\infty$, such that

$$
\int_{t}^{\infty}|g\{y(x)\}|^{2} d x \leqq M \int_{t}^{\infty}|y(x)|^{2} d x \text { for all } t \geqq T .
$$

Suppose further that $y(x)$ is a continuously differentiable solution of (4) which exists on the whole half-line $[0, \infty)$ and for which $y^{\prime \prime}(x)$ is integrable over every bounded interval and finite except at a finite or denumerable set of points. Then $y(x)$ cannot satisfy $(8)$ if $f(x)$ satisfies (3). 
(We have stated Theorem 5 in full for the reason given immediately before Theorem 2.)

Corollary 3. Under the conditions of Theorem 4 (or of Theorem 5) a solution $y(x)$ of $(4)$ which exists on the whole half-line $[0, \infty)$ cannot be an $L^{2}$ solution of (4) if $f(x)$ satisfies (3).

Corollary 3a. Under the conditions of Theorem 4 (or of Theorem 5), with condition (iv) of Theorem 2 (i.e. condition (iii) of Theorem 5) replaced by condition $\left(i v^{\prime}\right)(c f . \S 2)$, a solution $y(x)$ of (4) which exists on the whole half-line $[0, \infty)$ and is such that $y(x) \rightarrow 0$ as $x \rightarrow \infty$ cannot be an $L^{2}$ solution of $(4)$ if $f(x)$ satisfies (3).

We note that conditions (i) and (ii) of Theorem 4 may be relaxed in accordance with $\S 2$ and that condition (iv) of Theorem 2 (which occurs in Theorem 4) and condition (iii) of Theorem 5 may be replaced by condition (iv') (cf. § 2), in which case the validity of Corollary 3 becomes an open problem and we only have Corollary 3a. Further, we can obtain a theorem which is intermediate between Theorems 4 and 5, by replacing conditions (i) and (ii) of Theorem 4 by conditions (i), (ii) and (iii) of Theorem 3 (deleting the reference to the non-local existence of the solutions). Moreover, if we consider the above sequence of theorems we see that the most information about the functions $f(x)$ and $g(y)$ is required in Theorem 2 and the least in Theorem 5 (where, correspondingly, we require the most information about the given solution). Finally, since we can obtain Theorem 2 from Theorem 4 by imposing the additional condition (6) on $g(y)$, it is clear that Theorems 4 and 5 are nonvacuous. However we must verify that they are non-trivial, i.e. that there exist equations (4) which do not satisfy all the conditions of Theorem 2 (or 3) but which do have some solution defined on the whole half-line $[0, \infty)$ and other such equations which have solutions which become infinite for a finite value of $x$ (if this were not so the condition (6) would be unnecessary). We shall do this by producing examples which will show that the next theorem is non-vacuous.

Theorem 6. If $f(x)$ is continuous on $[0, \infty)$ and $y(x)$ is a continuous solution of the equation

$$
y^{\prime \prime}+f(x) y^{n}=0
$$

which is defined on the whole half-line $[0, \infty)$ then $y(x)$ cannot be an $L^{2 n}$ solution of (19) (i.e. a solution satisfying

if $f(x)$ satisfies (3).

$$
\left.0<\int_{0}^{\infty}|y(x)|^{2 n} d x<\infty\right)
$$

Proof. It is easy to verify that the function $f(y)=y^{n}$ satisfies the conditions of Theorem 4, except for condition (iv) of Theorem 2. But, by Lemma 1 (which does not depend on condition (iv) of Theorem 2) a solution $y(x)$ of (19) which satisfies (20) must be such that $y(x) \rightarrow 0$ as $x \rightarrow \infty$ and, for such a 
function, there exists a constant $T, 0 \leqq T<\infty$, such that $|y(x)| \leqq 1$ for $x \geqq T$. This means that $\int_{t}^{\infty}|y(x)|^{2 n} d x \leqq \int_{t}^{\infty}|y(x)|^{2} d x$ for all $t \geqq T$, which is simply (7) with $M=1$. Thus, the function $g(y)=y^{n}$ satisfies condition (iv') of Theorem 2 and this enables us to complete the proof, either by following the argument of $\S 4$ or by appealing to Theorem 4 .

Since $g(y)=y^{n}$ satisfies condition (iv') of Theorem 2, Theorem 6 and the proof of Corollary 1 immediately imply

Corollary 4a. If $f(x)$ is continuous on $[0, \infty)$ and $y(x)$ is a continuous solution of (19) which is defined on the whole half-line $[0, \infty)$ and is such that $y(x) \rightarrow 0$ as $x \rightarrow \infty$, then $y(x)$ cannot be an $L^{2}$ solution of $(19)$ if $f(x)$ satisfies (3).

We note that, by the discussion in $\S 2$, the function $f(x)$ in Theorem 6 and Corollary $4 \mathrm{a}$ may have a finite number of finite discontinuities. However, since $g(y)=y^{n}$ does not necessarily satisfy condition (iv) of Theorem 2, it is not clear whether or not we can relax the condition $y(x) \rightarrow 0$ as $x \rightarrow \infty$ in Corollary 4a. If we could, the result would be Corollary 4 , the analogue of Corollaries 1 , 2 and 3.

In conclusion, we present two examples. The first shows that an equation of the form (19) (i.e. of the form (4) but without condition (6)) can have a solution which exists on the whole half-line $[0, \infty)$. The second shows that such an equation can have a solution to a well-defined initial-value problem, which becomes infinite at a finite value of $x$. These examples show that Theorem 6 is non-vacuous and that Theorems 4 and 5 are non-trivial.

Example 1. The equation $y^{\prime \prime}-e^{-x} y^{2}=0$ has the solution $y(x)=e^{x}$ satisfying the initial conditions $y(0)=y^{\prime}(0)=1$. Here $f(x)=-e^{-x}$ satisfies (3) and $y(x)=e^{x}$ is not an $L^{4}$ solution (cf. Theorem 6) nor an $L^{2}$ solution (cf. Corollary 4a).

Example 2. The equation $y^{\prime \prime}+f(x) y^{2}=0$, where $f(x)=\left\{\begin{array}{l}-6,0 \leqq x \leqq 1 \\ -6 / x^{2+n}, x \geqq 1\end{array}\right.$ and $n>0$, has a unique solution satisfying the initial conditions $y(0)=1$, $y^{\prime}(0)=2$, namely the function $y(x)$ which is equal to $1 /(1-x)^{2}$ for $0 \leqq x<1$. Here $f(x)$ is continuous and satisfies (3) and the uniqueness of the solution satisfying the given initial conditions follows from the fact that (5) is satisfied. However, this solution becomes infinite at $x=1$ (and so we do not need to consider its form for $x>1$ ).

\section{REFERENCES}

(1) L. BIEBERBACH, Einführung in die Theorie der Differentialgleichungen im reellen Gebiet (Springer, Berlin, 1956).

(2) L. CesARI, Asymptotic Behavior and Stability Problems in Ordinary Differential Equations (Springer, Berlin, 1959). 
(3) R. Contı, Limitazioni " in ampiezza " delle soluzioni di un sistema di equazioni differenziali e applicazioni, Boll. Un. Mat. Ital. (3) 11 (1956), 344-349.

(4) R. Contı, Sulla prolungabilità delle soluzioni di un sistema di equazioni differenziali ordinarie, Boll. Un. Mat. Ital. (3) 11 (1956), 510-514. 1927).

(5) E. L. INCE, Ordinary Differential Equations (Longmans, Green, London,

(6) H. Kestelman, Modern Theories of Integration (University Press, Oxford, 1937).

(7) G. SANSONE, Equazioni differenziali nel campo reale, vol. 2 (Zanichelli, Bologna, 2nd ed., 1949).

(8) E. C. Titchmarsh, The Theory of Functions (University Press, Oxford, 2nd ed., 1939).

(9) A. WINTNER, The non-local existence problem of ordinary differential equations, Amer. J. Math. 67 (1945), 277-284.

(10) A. WINTNER, The infinities in the non-local existence problem of ordinary differential equations, Amer. J. Math. 68 (1946), 173-178.

(11) A. WINTNER, A criterion for the non-existence of $\left(\mathrm{L}^{2}\right)$-solutions of a nonoscillatory differential equation, J. London Math. Soc. 25 (1950), 347-351.

THE UNIVERSITY

Glasgow, W.2 